\title{
Multiphase isenthalpic flash: General approach and its adaptation to thermal recovery of heavy oil
}

Paterson, Duncan; Yan, Wei; Michelsen, Michael L.; Stenby, Erling H.

\section{Published in:}

AIChE Journal

Link to article, DOI:

10.1002/aic.16371

Publication date:

2019

Document Version

Peer reviewed version

Link back to DTU Orbit

Citation $(A P A)$ :

Paterson, D., Yan, W., Michelsen, M. L., \& Stenby, E. H. (2019). Multiphase isenthalpic flash: General approach and its adaptation to thermal recovery of heavy oil. AIChE Journal, 65(1), 281-293.

https://doi.org/10.1002/aic.16371

\section{General rights}

Copyright and moral rights for the publications made accessible in the public portal are retained by the authors and/or other copyright owners and it is a condition of accessing publications that users recognise and abide by the legal requirements associated with these rights.

- Users may download and print one copy of any publication from the public portal for the purpose of private study or research.

- You may not further distribute the material or use it for any profit-making activity or commercial gain

- You may freely distribute the URL identifying the publication in the public portal 


\title{
Multiphase Isenthalpic Flash: General Approach and its
}

\author{
Adaptation to Thermal Recovery of Heavy Oil \\ D. Paterson, W. Yan, M.L. Michelsen, E.H. Stenby \\ Technical University of Denmark
}

\begin{abstract}
Isenthalpic flash is a basic equilibrium calculation in process simulation. The recent interest in isenthalpic multiphase flash is mainly caused by the need for simulating thermal recovery of heavy oil. We present here systematic solutions to multiphase isenthalpic flash with full thermodynamics (such as EoS models) or with correlations for K-factors, and discuss how to tailor the general methods to systems encountered in thermal recovery of heavy oil. First, for the general situation with full thermodynamics we recommend a solution strategy which uses Newton's method for rapid convergence in the majority of cases and Q-function maximisation to safeguard convergence when Newton's method fails. The solution procedure is a generalisation of Michelsen's state function based two-phase flash to multiple phases. The general solution does not give special considerations for the components in the system and is not limited to the selected thermodynamic models and the number of phases. For thermal recovery processes where gas, oil and aqueous phases are typically involved, the stability analysis and initialisation steps are tailored to improve the efficiency. Second, since it is quite common in thermal reservoir simulators to describe phase equilibrium and heat properties with temperature dependent K-factors and separate correlations for heat capacities, we propose a formulation as an extension of the ideal solution isothermal flash formulation
\end{abstract}


to solve such problems. It uses a Newton-Raphson procedure to converge in the majority of cases and a nested loop procedure with the outer loop for a temperature search as a fallback approach for convergence. If the correlations for K-factors and for heat capacities are thermodynamically consistent, the outer loop can be treated as a maximisation. Finally, we present sytematic tests of the proposed algorithms using examples with full thermodynamics or K-factor based thermodynamics. The algorithms prove robust and efficient even in challenging cases including a narrow-boiling system, a degenerate system, and a four-phase system. The additional computational cost relative to the corresponding isothermal flash is modest and would be suitable for the purpose of thermal reservoir simulation.

\section{Introduction}

The phase equilibrium problem is at the heart of oil reservoir and chemical process simulation. The conventional example is isothermal flash with $(T, P, \boldsymbol{z})$ specified. The solution to the isothermal flash problem is at the global minimum of the Gibbs energy. The problem is usually solved by alternating use of stability analysis based on the tangent plane condition of

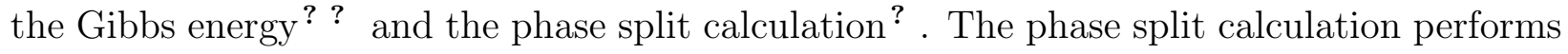
a local minimisation of the Gibbs energy and the stability analysis takes care of the global optimisation by searching for any new phase which can further reduce the Gibbs energy. A related equilibrium problem is the isenthalpic flash, with $(H, P, \boldsymbol{z})$ specified. The solution to the isenthalpic flash is the global maximum of the entropy. The same tangent plane stability analysis can be used to check if a new phase can be introduced.

The isenthalpic flash problem is relevant to a number of industrially important processes. It can be used to describe adiabatic expansion processes and steady state flow. There is renewed interest in its applicability to the simulation of thermal recovery of heavy oil, e.g., using steam injection? . For this thermal simulation it is necessary to add an energy balance to the system of equations used to carry out transient reservoir simulation. An additional 
variable must also be added, this is often chosen as either the temperature or the internal energy. When specifying the temperature, problems can be encountered for narrow boiling mixtures, as the energy and volume can change dramatically due to a small change in the temperature. Instead it can be preferable to use internal energy as a variable leading to an energy based flash (often isenthalpic flash) ${ }^{?}$.

Isenthalpic flash can be used with general thermodynamic models like equations of state. They provide a consistent and accurate description of fluid phase behaviour over a wide temperature and pressure range. There is a desire to use equations of state to simulate the production from oil reservoirs using thermal recovery techniques, especially when solvents are used in combination with steam injection. However, flash calculation with an equation of state is more complex and can become a bottleneck in the simulation speed. As a trade-off, one can limit the number of grid blocks in a simulation. Most commercial thermal reservoir simulators (e.g. CMG STARS) generally use temperature dependent K-value correlations instead of an equation of state. It is also common to use a correlation for the residual enthalpy of the liquid phases, which is not always thermodynamically consistent with the employed K-value correlation. The system in such a situation reduces to an ideal solution where a simplified procedure can be used.

Most early work on the isenthalpic flash problem focused on calculations for two phases (e.g. $\left.{ }^{?}\right)$. The first fully multiphase implementation was described by ${ }^{?}$, where both a first order direct substitution approach (with acceleration? ) and a second order approach were presented. The direct substitution algorithm has been investigated by a number of authors. Alternatives to the acceleration were suggested by ${ }^{?}$ along with a combined approach using direct substitution and a nested isothermal flash. Carrying out simultaneous phase split and stability analysis calculations was presented by ${ }^{?}$ as an alternative to the sequential approach to the phase split calculation and stability analysis, this was further examined by ${ }^{\text {. }}$ Narrow boiling fluids were investigated by ${ }^{\text {? }}$ who suggested using bisection for degenerate cases. $^{?}$ used the negative flash to avoid stability analysis. For nearly ideal mixtures direct 
substitution is rapidly convergent, however the rate of convergence is slow for non-ideal mixtures and may even become divergent. Large scale simulation may involve millions of separate flash calculations. The linear rate of convergence and lack of a guarantee of convergence can cause unacceptable computational time or complete failures. A robust approach is required and a more rapid second order approach desired. One possible solution is the direct maximisation of the entropy. This was used by ${ }^{\text {? }}$ for reservoir simulation with a robust implementation developed by ${ }^{?}$ where the cost was compared to isothermal flash. demonstrated a general solution strategy for two-phase isenthalpic flash, as well as several other state function based flash specifications. He proposed to use a Newton approach for efficiency with a Q-function maximisation approach for robustness. The Q-function maximisation approach is essentially a nested loop approach with an isothermal flash in the inner loop and an outer loop maximising the Q-function to determine the temperature. The Q-function maximisation is used as a backup when direct solution of the equilibrium equations by the Newton approach fails.

In this work we extend the two-phase work of ${ }^{?}$ to the general multiphase isenthalpic flash. For the Q-function maximisation method, challenging cases are highlighted and solutions proposed. A full algorithm is described for the general solution of the multiphase isenthlpic flash problem, capable of dealing with narrow boiling and degenerate cases and dynamically adding and removing phases. For thermal recovery of heavy oil, the general algorithm is tailored by including a separate phase for initialisation and using the Rachford-Rice type equations to improve early steps in the partial Newton method with poor initial estimates. For commercial thermal recovery simulators, temperature dependent K-factors and separate correlations for heat capacities are typically used. These correlations correspond to an ideal solution model. For this case, we propose a formulation as an extension of the isothermal flash

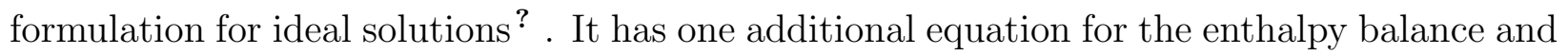
is solved by the Newton approach in the majority of cases. A nested loop procedure with the outer loop searching for temperature is used as a fallback method. It should be noted that 
the ideal solution model employed in commercial simulators is not always thermodynamically consistent. If the correlations for K-factors and for heat capacities are consistent, the outer loop can be treated as a maximisation.

In the following sections, we first present how to solve multiphase isenthalpic flash for K-factor based thermodynamics and for full thermodynamics using different methods, including the ideal solution method for the former and the partial Newton, Newton and Qmaximisation methods for the latter. We then present how to implement each method and discuss how to handle specific issues like heavy oil-water systems in thermal recovery. We finally present seven multiphase isenthalpic flash examples for both the ideal solution case and with general equation of state based thermodynamics. Each example is tested over a wide pressure and molar enthalpy range so as to evaluate the robustness and efficiency of the proposed methods. The computational cost of the isenthalpic flash is compared with that of the isothermal flash at the same conditions (the specified pressure and converged temperature) showing that the additional cost would not be prohibitive for reservoir simulation.

\section{Isenthalpic flash}

For a mixture of $C$ components with molar amounts $\left\{z_{i}\right\}$ at pressure $P^{s p e c}$ and enthalpy $H^{\text {spec }}$ the aim of isenthalpic flash is to find the number of phases $F$, with phase fractions $\left\{\beta_{j}\right\}$, the composition of each phase $\left\{y_{i j}\right\}$ and the equilibrium temperature $T$. The solution corresponds to the global maximum in the entropy:

$$
\max \frac{S(H, P, \boldsymbol{n})}{R}
$$

subject to the material balance and enthalpy constraints

$$
\sum_{j=1}^{F} n_{i, j}-z_{i}=0, \quad \forall i
$$




$$
\sum_{j=1}^{F} H_{j}-H^{\text {Spec }}=0
$$

and non-negativity of any component in any phase

$$
n_{i, j} \geq 0, \quad \forall i, j
$$

Direct entropy maximisation was investigated by ${ }^{?}$ and ${ }^{?}$. However thermodynamic models are commonly solved at a given $(T, P, \boldsymbol{n})$ for each phase rather than $(H, P, \boldsymbol{n})$. The enthalpy is then described as a constraint which is nonlinear in the variables. Most implementations of isenthalpic flash carry out some form of constrained maximisation, or simply solve for the equilibrium conditions, with the enthalpy specification met at the solution.

For any intermediate solution of $F$ phases, stability analysis ${ }^{?}$ can check if an additional phase should be introduced and provide an initial composition estimates for the new phase. A phase split step is subsequently performed to determine the new equilibrium distribution at the specified pressure and enthalpy. In this study we employ essentially the same stability analysis and mainly focus on the variations in the other parts of the flash procedure.

The methods used for isenthalpic flash depend on whether the thermodynamic model is a full equation of state or a set of correlations for K-factors. Table 1 presents an overview of the methods discussed in this study. For thermal recovery calculation where the system involves an aqueous phase, we can adapt the general methods by making some of the steps more efficient. No such adaptation is needed if only K-factor correlations are used.

\section{Ideal solution}

In many simulations a full equation of state is not used. Instead correlations are used to evaluate both the K-values and the enthalpy of the liquid phases (or enthalpy of vaporisation). The solution procedure for these ideal solutions is simpler than when using full thermodynamics. 
In the ideal solution approximation it is assumed that the fugacity coefficient for each component in each phase is available as a function of temperature and pressure $\hat{\varphi}_{i, j}(T, P)$. The residual enthalpy of the phases must also be available using a suitable correlation. Using the fugacity coefficients, the composition of each phase is found as:

$$
y_{i, j}=\frac{z_{i}}{E_{i} \hat{\varphi}_{i, j}} \quad E_{i}=\sum_{k=1}^{F} \frac{\beta_{k}}{\hat{\varphi}_{i, k}}
$$

with temperature derivatives

$$
\begin{gathered}
\frac{\partial y_{i, j}}{\partial T}=y_{i, j}\left(-\frac{\partial \ln \hat{\varphi}_{i, j}}{\partial T}-\frac{\partial \ln E_{i}}{\partial T}\right) \\
\frac{\partial \ln E_{i}}{\partial T}=-\frac{\sum_{k}^{F} \beta_{k} y_{i k} \frac{\partial \ln \hat{\varphi}_{i, k}}{\partial T}}{z_{i}}
\end{gathered}
$$

and phase fraction derivatives

$$
\frac{\partial y_{i, j}}{\partial \beta_{k}}=-\frac{y_{i k} y_{i j}}{z_{i}}
$$

The system of equations which must be satisfied at equilibrium is:

$$
g_{j}=1-\sum_{i}^{C} y_{i j} \quad j=1,2, \ldots, F
$$

and

$$
g_{F+1}=H-H^{s p e c}
$$

The enthalpy is found from:

$$
H=\sum_{j=1}^{F} \beta_{j} \sum_{i=1}^{C} y_{i, j} h_{i, j}=\sum_{j=1}^{F} \beta_{j} \sum_{i=1}^{C} y_{i, j}\left(h_{i, j}^{I G}+h_{i, j}^{r}\right)
$$


The Jacobian for this system of equations is

$$
\begin{gathered}
J_{j k}=-\sum_{i=1}^{N C} \frac{\partial y_{i, j}}{\partial \beta_{k}} \\
J_{j, F+1}=-\sum_{i=1}^{C} \frac{\partial y_{i, j}}{\partial T} \\
J_{F+1, j}=\sum_{i=1}^{C} y_{i, j} h_{i, j}+\sum_{k=1}^{F} \beta_{k} \sum_{i=1}^{C} h_{i, k} \frac{\partial y_{i, k}}{\partial \beta_{j}}
\end{gathered}
$$

the ideal gas terms cancel in the difference in equation 11 to leave:

$$
J_{F+1, j}=\sum_{i=1}^{C} y_{i, j} h_{i, j}^{r}+\sum_{k=1}^{F} \beta_{k} \sum_{i=1}^{C} h_{i, k}^{r} \frac{\partial y_{i, k}}{\partial \beta_{j}}
$$

and finally

$$
J_{F+1, F+1} \approx \frac{1}{R} \sum_{j=1}^{F} \beta_{j}\left(C_{P, j}+\sum_{i=1}^{C} h_{i j} \frac{\partial y_{i j}}{\partial T}\right)
$$

This approximation is exact at the solution. If the temperature derivatives of the component enthalpies are readily accessible from the equation used then it may be replaced with:

$$
J_{F+1, F+1}=\sum_{i=1}^{C} \sum_{j=1}^{F}\left(n_{i j} \frac{\partial h_{i, j}}{\partial T}+\beta_{j} h_{i, j} \frac{\partial y_{i j}}{\partial T}\right)
$$

If the thermodynamic model employed is consistent then the temperature derivative of the fugacity and the residual heat capacity are related through:

$$
R T^{2} \frac{\partial \ln \hat{\varphi_{i j}}}{\partial T}=-h_{i, j}^{r}
$$

In which case equation 12 can be replaced by

$$
J_{F+1, j}=R T^{2} J_{j, F+1}
$$


Alternatively a symmetric system of equations can be arrived at by dividing equation 7 by $R T^{2}$.

The presented system of equations and Jacobian will be convergent in the majority of cases. However in some cases it is necessary to nest the isothermal flash in an inner loop with the temperature updated in the outer loop.

\section{Newton's method}

When each phase is represented using an equation of state the ideal solution may no longer be appropriate. Instead the fugacity coefficients are now also functions of composition $\hat{\varphi}_{i, j}\left(T, P, \boldsymbol{n}_{j}\right)$. The equilibrium equations and enthalpy constraint can be solved directly using Newton's method. For each component, set its mole number in a certain phase as dependent through the material balance:

$$
n_{i, J(i)}=z_{i}-\sum_{j \neq J(i)}^{F} n_{i, j} \quad \forall i
$$

where $J(i)$ represents the phase for component $i$ where the component is present in the greatest amount. The equilibrium equations are

$$
g_{l}=\ln \hat{f}_{i, j}-\ln \hat{f}_{i, J(i)}, \quad l=1,2, \ldots, C(F-1),
$$

$$
i=1,2, \ldots, C, j=1,2, \ldots, F, j \neq J(i)
$$

and the enthalpy constraint

$$
g_{C(F-1)+1}=\frac{H^{s p e c}-H}{R T}
$$


These equations lead to a symmetric Jacobian with elements

$$
\begin{array}{r}
J_{l, o}=\left(\delta_{j, m}-\delta_{j, J(k)}\right) \frac{\partial \ln \hat{f}_{i, j}}{\partial n_{k, m}}+\left(\delta_{J(i), J(k)}-\delta_{J(i), m}\right) \frac{\partial \ln \hat{f}_{i, J(i)}}{\partial n_{k, J(i)}} \\
l=1,2, \ldots, C(F-1), o=1,2, \ldots, C(F-1), i=1,2, \ldots, C, k=1,2, \ldots, C \\
j=1,2, \ldots, F, m=1,2, \ldots, F, j \neq J(i), m \neq J(k)
\end{array}
$$

with $\delta_{j, m}$ representing the Kronecker delta function. The derivative of the fugacity is:

$$
\frac{\partial \ln \hat{f}_{i, j}}{\partial n_{k, j}}=\frac{\delta_{i, k}}{n_{i, j}}-\frac{1}{\beta_{j}}+\frac{\partial \ln \hat{\varphi}_{i, j}}{\partial n_{k, j}}
$$

The remaining row and column are

$$
J_{C(F-1)+1, l}=J_{l, C(F-1)+1}=T\left(\frac{\partial \ln \hat{f}_{i, j}}{\partial T}-\frac{\partial \ln \hat{f}_{i, J(i)}}{\partial T}\right)
$$

with the final equation:

$$
J_{C(F-1)+1, C(F-1)+1}=-\frac{C_{P}}{R}
$$

The variables used for this Jacobian are $\Delta \boldsymbol{n}_{j}, \Delta \ln T$. This is the extension of the implementation of ${ }^{?}$ to the multiphase case. Alternatively it is possible to use component yields $\theta_{i, j}=n_{i, j} / z_{i}$ in place of mole numbers as variables as shown by ${ }^{?}$. This may lead to a better conditioned Jacobian but the resulting convergence behaviour is similar.

\section{Partial Newton}

The full Newton method often requires an accurate initial estimate. For the isothermal flash successive substitution using the multiphase Rachford-Rice equations is commonly carried out for a small number of iterations. Similarly for isenthalpic flash it is possible to use direct substitution, this is similar to successive substitution but due to the temperature dependence of the fugacity coefficients, they must be updated at each iteration. 
From the ideal solution equations given here it is possible to obtain the direct substitution equations by setting one phase fraction as dependent through the material balance

$$
\beta_{F}=1-\sum_{j=1}^{F-1} \beta_{j}
$$

and replacing equation 6 with:

$$
g_{j}=\sum_{i=1}^{C}\left(y_{i F}-y_{i j}\right) \quad j=1,2, \ldots, F-1
$$

The Jacobian of equations 23 and 7 with one phase fraction set as dependent can be found and will be similar to that presented by ${ }^{?}$. The direct substitution method is often useful for early iterations, and can be used to easily remove phases from the system of equations.

An alternative partial Newton method is possible starting from the full Newton method. Making an ideal solution approximation, the composition derivative of the fugacity coefficient can be set to zero:

$$
\frac{\partial \ln \hat{\varphi}_{i, j}}{\partial n_{k, j}}=0
$$

Otherwise the system of equations and Jacobian is the same as presented for Newton's method in equations 20 to 22 . This leads to a simple Jacobian where the explicit inverse is readily available for part of the matrix for the two-phase case, reducing the number of variables from $C+1$ to 2 . For the multiphase case a similar reduction in variables is possible though more complex.

The partial Newton method will be convergent when the mixture is not highly nonideal $^{?}$. However the rate of convergence can be intolerably slow, with a nested isothermal flash often outperforming the computational cost of the partial Newton method alone.

The update from the Newton-Raphson iteration is only convergent to the desired solution with a suitably close initial estimate. Often non-convergent updates can be encountered when more than one eigenvalue from the Newton update is negative. In these cases a small 
number of additional partial Newton steps can be useful, or a reduction of the impact of the composition derivatives of the fugacity coefficient using a suitable scaling factor can aid in convergence (i.e. instead of setting the derivatives to zero, equation 24 , reduce them by a half). Doing so for a small number of iterations can help with the convergence while not significantly increasing the computational cost of the method.

\section{Q-function maximisation}

? demonstrated that using a nested loop for $(P, T)$ flash, the $(P, H)$ flash problem could be posed as a maximisation of a suitable objective function:

$$
Q=\frac{G_{\min }(T)-H^{\text {spec }}}{R T}
$$

with the gradient

$$
\frac{\partial Q}{\partial \frac{1}{T}}=\frac{H-H^{s p e c}}{R}
$$

and the Hessian

$$
\frac{\partial^{2} Q}{\partial\left(\frac{1}{T}\right)^{2}}=-\frac{T^{2} C_{p, \min }}{R}
$$

with

$$
C_{p, \min }=\sum_{j=1}^{F} \beta_{j} C_{p, j}+\sum_{i=1}^{C} \sum_{j=1}^{F}\left(\frac{\partial H_{j}}{\partial n_{i, j}}-\frac{\partial H_{F}}{\partial n_{i, F}}\right) \frac{\partial n_{i, j}}{\partial T}
$$

where the first term is the pure phase heat capacity and the second is the heat capacity of phase change. The Hessian requires the solution to the flash equations and is always negative.

Instead of evaluating the Hessian directly, it is possible to use the Jacobian presented in equations 20 - 22 to evaluate the same temperature step. The step in the component amounts can be used as an initial estimate for the next nested isothermal flash iteration. The convergence in the temperature is quadratic, and using a robust and efficient isothermal flash solver the Q-function maximisation is efficient. 


\section{Modifications for thermal simulation including an aque-}

\section{ous phase}

Simulation of oil production through the use of thermal methods (e.g. steam injection) is an important area of application for isenthalpic flash. There are a number of issues commonly encountered in such simulations which are addressed in this section. Firstly the case of a discontinuity in isothermal flash (when there are more phases than components), this can occur frequently close to a steam injector and requires special treatment during simulation. Furthermore there are often a number of components which are present only in trace amounts in the aqueous phase, the solubility of these components can change by many orders of magnitude as the temperature changes which can significantly hinder convergence.

The case where there are more phases than components is of obvious concern since it does not exist when using $(P, T)$ flash. Therefore using temperature as a variable is not possible and variable substitution or isenthalpic flash must be used. Using a nested isothermal flash is susceptible to oscillations if $F>C$. Similarly at the solution when $F>C$ the Gibbs energy of both stable roots to the equation of state can be identical for one or more phases and may lead to the wrong root being chosen.

There are a number of possible solutions to deal with this problem. For Newton's method and the partial Newton methods, selecting the desired root to the equation of state is possible in a manner proposed by ${ }^{\text {? }}$. For Q-function maximisation it would be necessary to find the transition temperature then split the two oscillating phases to meet the enthalpy constraint and material balance. This will require a few additional iterations to find the transition temperature.

An alternative which will work with both Q-function maximisation and Newton's method (or a partial Newton method) is to introduce a tolerably small amount of an additional component (e.g. $10^{-8}$ ). This will remove the discontinuity in the enthalpy though the use of Q-function maximisation may have issues due to the highly narrow boiling nature of the 
new mixture.

For initialisation of the vapour liquid equilibrium a simple K-value correlation is often used (for example? ${ }^{\text {p }}$ proposed the use of the Wilson K-factors). This can be used with a second correlation for the solubility of water in oil allowing for a three phase initialisation. The solubility of water in the oleic phase was modelled based on the correlation of ${ }^{\text {, }}$, where the fugacity coefficient for each phase is calculated as:

$$
\begin{gathered}
\hat{\varphi}_{i}^{v}=1 \forall i, \quad, \hat{\varphi}_{i}^{l}=K_{i}^{\text {Wilson }} \quad i \neq w, \quad \hat{\varphi}_{w}^{a}=K_{w}^{\text {Wilson }} \\
\hat{\varphi}_{w}^{l}=\exp \left(\ln K_{w}^{\text {Wilson }}+21.263-0.0595 T+0.0000408 T^{2}\right), \quad \hat{\varphi}_{i}^{a}=10^{10}, \quad i \neq w
\end{gathered}
$$

The residual enthalpy of each phase was evaluated using equation 15 . The ideal solution method given above was used to solve this ideal solution problem. In general the initial estimates provide reasonably accurate temperature and phase composition estimates which can be used with Newton's method following only a small number of partial Newton steps. More complex correlations are possible for the solubility of components in the aqueous phase, though often their solubility is very small and a large constant value is suitable.

The stability analysis for such systems can also be simplified. Instead of using each pure component as an initial estimate it is generally sufficient to use the water component, lightest component, heaviest component and an intermediate, to initialise the stability analysis. A general procedure for doing so is described by ${ }^{?}$.

Though the initial estimate from the initialisation is often close to the solution temperature, the molar amount of some components in the aqueous phase may be far from the solution. When using the partial Newton method such deviations can lead to very slow rates of convergence, the direct substitution method directly calculates the composition of each phase based on the fugacity coefficients and does not run into such difficulties. 
The partial Newton method (and the full Newton method) can be improved in the early steps by using an update based on the Rachford-Rice equations. Once the partial Newton update is found $\left(\Delta \boldsymbol{n}_{j}, \Delta T\right)$, the change in the dependent mole number of component $i$ can be found as

$$
\Delta n_{i, F}=-\sum_{j=1}^{F-1} \Delta n_{i, j}
$$

and the update to the equilibrium K-factors evaluated:

$$
\Delta \ln K_{i, j}=\frac{\Delta n_{i, j}}{n_{i, j}}+\frac{\sum_{i=1}^{C} \Delta n_{i, F}}{\beta_{F}}-\frac{\Delta n_{i, F}}{n_{i, F}}-\frac{\sum_{i=1}^{C} \Delta n_{i, j}}{\beta_{j}}
$$

For each component where the fugacity difference between the phases is large (e.g. if $\mid \ln \hat{f}_{i, j}-$ $\ln \hat{f}_{i, F} \mid>1$ ), an estimate for the new molar amount can be evaluated from

$$
n_{i, j}=\frac{\beta_{j} K_{i, j} z_{i}}{1+\sum_{j=1}^{F-1} \beta_{k}\left(K_{i, k}-1\right)}
$$

where the values for $\beta_{j}$ have been updated as $\beta_{j}=\beta_{j}^{\text {old }}+\sum_{i=1}^{C} \Delta n_{i, j}$. For component $i$ the dependent phase is found

$$
n_{i, F}=\frac{\beta_{F} z_{i}}{1+\sum_{j=1}^{F-1} \beta_{k}\left(K_{i, k}-1\right)}
$$

And the flow of component $i$ is then rescaled by $z_{i}$.

$$
n_{i, j}=\frac{z_{i} n_{i, j}}{\sum_{j=1}^{F} n_{i, j}}
$$

This update ensures the poor initial estimate of the hydrocarbon components in the aqueous phase do not cause significant convergence issues. Though described for updating only the components in the aqueous phase the general method is sometimes useful following large temperature changes where the solubility of some components in a near pure aqueous phase can change by one or more orders of magnitude (in either the full or partial Newton implementations). 
The update can also provide an estimate for the composition of a phase which might be removed:

$$
y_{i, j}=\frac{K_{i, j} z_{i}}{1+\sum_{j=1}^{F-1} \beta_{k}\left(K_{i, k}-1\right)}
$$

This composition estimate can be useful either to attempt to re-introduce the phase or as an initial estimate for stability analysis. In simulation if the deleted phase is close to the phase boundary it will provide an excellent initial estimate to be stored for stability analysis skipping using the shadow region method of ?

\section{Implementation}

A number of different implementations of isenthalpic flash have been presented. These can be split between those which use K-factor approximations (ideal solution) and full thermodynamics (Newton's method, partial Newton, and Q-function maximisation). For each of these a suitable implementation is necessary.

\section{Ideal solution}

Given an initial estimate with $F$ phases at temperature $T$ one iteration of the ideal solution procedure is carried out as:

1. For each phase evaluate the thermodynamic properties

$$
\ln \hat{\varphi}_{i, j}, \quad \frac{\partial \ln \hat{\varphi}_{i, j}}{\partial T}, \quad h_{i, j}, \quad C_{p, j}
$$

2. Calculate the composition of each phase

$$
y_{i, j}=\frac{z_{i}}{E_{i} \hat{\varphi}_{i, j}} \quad E_{i}=\sum_{k}^{F} \frac{\beta_{k}}{\hat{\varphi_{i, k}}}
$$

and the temperature derivatives from equations 3 and 4. 
3. Evaluate the gradient from equations 6 and 7 and the Jacobian from equations 9 - 13 .

4. Factorise and solve the system of equations to find the update to the phase fractions and temperature

$$
(\Delta \boldsymbol{\beta}, \Delta T)^{T}=-\boldsymbol{J}^{-1} \boldsymbol{g}
$$

5. Evaluate the new phase fractions $\boldsymbol{\beta}_{\text {new }}=\boldsymbol{\beta}+\alpha \Delta \boldsymbol{\beta}$ and temperature $T_{\text {new }}=T+\alpha \Delta T$. If any phase fraction becomes negative then reduce the step length modifier so one phase is set to zero. Limiting the step in temperature may be necessary to avoid stepping outwith the bounds on the ideal gas heat capacity correlation, normally $\alpha=1$.

6. If no phase has been deleted then check for convergence and output the result.

7. If a phase is removed then iterations can continue with the remaining phases. It can be checked if the deleted phase can be re-introduced. If $\Delta \beta>0$ for the removed phase then it can be re-introduced and iterations continued.

The proposed method is useful for systems where the enthalpy and fugacity coefficients are represented using separate models. If the proposed method is not convergent for the ideal solution case, it is necessary to nest an isothermal flash. The isothermal flash problem can be solved using the gradient and Hessian (equations 6 and 9) of the equation

$$
Q=\sum_{j=1}^{F} \beta_{j}-\sum_{i=1}^{C} z_{i} \ln E_{i}
$$

using the algorithm described by ${ }^{\text {? }}$ chapter 11 for solving the multiphase ideal solution phase split of ${ }^{?}$.

One example of an ideal solution model is the Wilson K-factor approximation:

$$
K_{i}^{W i l s o n}=\frac{P_{c, i}}{P} \exp \left(5.373\left(1+\omega_{i}\right)\left(1-\frac{T_{c, i}}{T}\right)\right)
$$


The vapour phase can be assumed to be an ideal gas $\hat{\varphi}_{i}^{v}=1$ and the liquid phase fugacity is equal to the Wilson K-factor. The temperature derivative can then be evaluated.

\section{Partial Newton}

Two partial Newton methods have been mentioned. The first is direct substitution and the second is a partial Newton method based on the full Newton method with the composition derivatives of the fugacity coefficients assumed to be zero. The implementation for direct substitution can follow the procedure described by ${ }^{\text {. }}$.

The partial Newton method described as a simplification of the full Newton method can be carried out as:

1. For each phase evaluate the thermodynamic properties

$$
\ln \hat{\varphi}_{i, j}, \quad \frac{\partial \ln \hat{\varphi}_{i, j}}{\partial T}, \quad H_{j}, \quad C_{p, j}
$$

2. Evaluate the gradient and Jacobian, then using the properties of the Jabocian solve for $\Delta \boldsymbol{n}, \Delta \ln T$.

3. For each phase find $\Delta \beta_{j}=\sum_{i=1}^{C} \Delta n_{i, j}$. If for any phase $-\Delta \beta_{j}>\beta_{j}$ then it may be necessary to remove that phase. To do so it will be necessary to use equations 31-35 to avoid violating the material balance.

4. If for a component $i$ there is a very large difference in the fugacity between phases (e.g. $\left.\left|\ln \hat{f}_{i, j}-\ln \hat{f}_{i, F}\right|>1\right)$ then for that component use equations 31-35 to update molar amount of that component in each phase.

5. After taking a step, if a phase is deleted, then use its composition estimate from 35 in the next iteration. If at the next step $\Delta \beta_{j}<0$ for the deleted phase, then it should be removed from the system of equations, otherwise it can be re-introduced. 
6. If following a step any of the phases are oscillating between vapour and liquid it indicates that a new phase must be introduced. If there is no vapour phase in the current mixture one can be introduced as an ideal gas (i.e. $\hat{\varphi}_{i, j}^{I G}=1$ ). Otherwise if a liquid must be introduced, stability analysis can be attempted, if not successful then a switch to Q-function maximisation is necessary.

Often it is best to take only a small number of partial Newton steps before switching to a full Newton method. In the given implementation 5 steps of partial Newton were used before a switch to the full Newton method. One advantage of the partial Newton method is that it is likely to under-predict the step-size, and therefore it is often safe to remove a phase. The convergence of direct substitution and that of the partial Newton method are similar and either can be used.

\section{Newton's method}

One iteration with the full Newton method can be taken as:

1. For each phase evaluate the thermodynamic properties

$$
\ln \hat{\varphi}_{i, j}, \quad \frac{\partial \ln \hat{\varphi}_{i, j}}{\partial n_{k, j}}, \quad \frac{\partial \ln \hat{\varphi}_{i, j}}{\partial T}, \quad H_{j}, \quad C_{p, j}
$$

2. For each component define the phase present in the greatest amount as dependent through the material balance. The components in the remaining phases are the independent variables.

3. Evaluate the system of equations defined by equations 18 and 19 and the Jacobian defined in equations 20-22.

4. Factorise of the Jacobian. If more than one eigenvalue is negative then it may indicate there will be convergence issues, the step can be attempted or can be damped by reducing the influence of the partial derivatives of the fugacity coefficients. In the 
presented implementation the derivatives were reduced by half once if more than one eigenvalue was negative, though there is little benefit compared to using more partial Newton steps.

5. Evaluate the step. For each phase evaluate $\Delta \beta_{j}=\sum_{i=1}^{C} \Delta n_{i, j}$. If for any phase $-\Delta \beta_{j}>\beta_{j}$ then it may be necessary to remove the phase. If the phase is present in only a small amount (e.g. $\left.\beta_{j}<0.01\right)$ then a partial Newton step is used to ensure that the phase can be safely removed. Otherwise if the phase is present in a significant amount then it is possible the step is too large, using a step length modifier the step should be taken, ensuring that no component becomes negative.

6. Check for convergence. If converged output the result. If the deviation in enthalpy has increased significantly it is likely the step has been too large, apply a step length modifier $(\alpha=1 / 3$ used in this implementation) and attempt the step again.

7. If it is clear that the solution is oscillating or there are excessive steps in temperature or phase fraction then a switch to Q-function maximisation may be necessary. In the presented implementation the maximum number of iterations taken before a switch to Q-function maximsiation was $(F+1) \times 10$, where $F$ is the current number of phases.

\section{Q-function maximisation}

The use of a nested isothermal flash is often more costly than direct Newton's method for isenthalpic flash, however the cost can be minimised by using Q-function maximisation. Two implementations of Q-function maximisation are possible, one where stability analysis is only used at the local maximum of the Q-function, and a second where stability analysis is used at each iteration to ensure the global minimum of the Gibbs energy is found, this is more costly but necessary for some difficult cases. In general stability analysis is carried out at each step only when it is clear there are issues with convergence, in this implementation if a phase was introduced or removed multiple times, or after 10 iterations (where an iteration 
is the evaluation of the Newton step in temperature), stability analysis was used at each iteration. For example given a mixture of $F$ phases one iteration is taken as

1. Carry out isothermal flash using the $F$ phases to find the local minimum in Gibbs energy. Optionally carry out stability analysis with isothermal flash to find the global minimum.

2. Evaluate the Q-function from equation 25. Evaluate the Newton step from the Gradient and Hessian or from equations 18 - 22. Take the step in temperature and update the composition.

3. At the new temperature solve the isothermal flash using the $F$ phases to find the local minimum in Gibbs energy. Optionally carry out stability analysis with isothermal flash to find the global minimum.

4. Re-evaluate the Q-function, if it has increased then accept the step and check for convergence, otherwise use a line search in temperature until the Q-function has increased.

5. If convergened then carry out stability analysis if it has not yet been checked.

Stability analysis is a computationally expensive element of isothermal flash calculations, in particular when multiple phases are involved. In general it is best to avoid it when possible. However in a number of cases it is necessary to carry out stability analysis multiple times when maximising the Q-function.

The enthalpy and heat capacity of example 1 at 1 bar is presented in figure 1 . When close to a phase boundary the heat capacity of phase change in equation 28 increases rapidly, with a discontinuity at the boundary. This can lead to poor convergence when using a Q-function maximisation in the region close to the phase boundary, and is the problematic region when carrying out reservoir simulation with temperature as a variable. An overstep leading to the removal of a phase can lead to increase in the Q-function (and Gibbs energy), even if the 
phase should be present at equilibrium. Often close to a phase boundary it is necessary to carry out stability analysis at multiple iterations to avoid the trivial solution.

Another case where it may be necessary to carry out stability analysis before reaching a local solution is demonstrated in figure 2. This figures shows the enthalpy of the LLE, VLE and VLLE solutions to the isothermal flash for example 1 at 1 bar.

In the range between $-6700 K<H / R<-5175 K$ it is not possible to find a solution using only two phases. It is necessary to introduce a third phase (aqueous). Given only two phases the solution will oscillate between the VLE and LLE solutions. In some cases when using the partial Newton or full Newton method an intermediate two-phase solution can be found with only two phases, though one phase will be intrinsically unstable.

\section{Results}

We deal with a number of examples in the results section. These are summarised in table 2. Each mixture is modelled as either an ideal solution (example 1), using the Peng Robinson (PR) equation of state? or the SRK equation of state? . A detailed description of each mixture is given in the appendix.

For the ideal solution we have only a single methodology to solve the isenthalpic flash problem given in the implementation. This was limited to using only 25 iterations before a backup nested loop approach was used. To demonstrate the convergence of the ideal solution isenthalpic flash implementation example 1 is considered with the Wilson K-factor approximation (assuming only two phases), with the residual enthalpy found from equation 15. Figure 3 shows the quadratic convergence behaviour of the ideal solution method, the conditions used are $H / R=-4000 \mathrm{~K}$ and $P=20 \mathrm{bar}$. The initial estimate is $T=300 \mathrm{~K}$ and $\beta=0.5$ the solution conditions are $T=511.2 \mathrm{~K}$ and $\beta=0.345$. The initial estimate is very poor yet convergence is still obtained rapidly. The vapour phase is removed on the first iteration, then re-introduced on the fourth iteration. 
To further demonstrate the efficacy of the ideal solution approximation, a pressure range of 1 bar $<P<100$ bar and enthalpy range of $-6000 \mathrm{~K}<H / R<5000 \mathrm{~K}$ was flashed with a step size of $5 \mathrm{~K}$ and $0.1 \mathrm{bar}$. A third water phase was introduced through the use of equations 29 and 30 . The initial estimate was set at $T=300 \mathrm{~K}$ and $\beta_{j}=1 / 3$ for all three phases. The number of iterations (where an iteration is counted as factorising the system of equations) to obtain convergence to a tolerance of $|g|_{\infty}=10^{-10}$ using the isothermal flash algorithm of ${ }^{?}$ and the isenthalpic flash presented here are plotted in figures $4 \mathrm{a}$ and $4 \mathrm{~b}$ respectively.

Figure 4 shows that in most of the region scanned the number of iterations necessary is very small, this is despite a constant initialisation temperature of $T=300 \mathrm{~K}$ which is often very far from the solution. Problems are encountered when close to the critical temperature of each component. This is noticeable on figure 4 where an increase in the number of iterations follows the isotherms close to the critical temperature of each component. The cause of this is the model used for the residual enthalpy which decreases to zero at the critical temperature (causing a discontinuity in the heat capacity). Equation 37 is used to calculate the heat of vaporisation for example 1.

$$
\frac{H^{\text {vap }}}{R}=\left\{\begin{array}{lr}
C_{1} \times\left(1-T_{r}\right)^{\left(C_{2}+C_{3} T_{r}+C_{4} T_{r}^{2}\right),} & \text { if } T<T_{c} \\
0, & T \geq T_{c}
\end{array}\right.
$$

The parameters for the equation are given in the appendix. When close to the critical temperature there is an almost discontinous change in the enthalpy of vaporisation which leads to the streaked, near vertical, lines in figure $4 \mathrm{~b}$, each of these lines corresponds to the critical temperature of one component in the mixture.

The ideal solution isenthalpic flash used approximately 3.3 times as much computational time as the multiphase Rachford-Rice equations. Using only a nested isothermal flash for isenthalpic flash, the cost was 4.8 times more than the isothermal flash. The large increase in the cost of isenthalpic flash is due to the repeated evaluation of the fugacity coefficients and enthalpy at each iteration and initial estimate of temperature being far from the solution. 
Isothermal flash only requires a single evaluation of the fugacity coefficients. When using the ideal solution model, the flash is only a small contribution to the overall simulation cost and the proposed method should be sufficient. Using an equation of state takes more than 20 times as long.

For the remaining examples an equation of state is used to evaluate the fugacity coefficients and residual enthalpy. For compositional reservoir simulation the flash calculations may limit the size of the reservoir simulation. For isenthalpic flash to be viable it is necessary that the increase in computational cost is not prohibitive when compared to isothermal flash. For each of the examples a large region of the phase envelope was scanned and the time to carry out an isenthalpic flash recorded. At the solution temperature the problem was re-initialised and an isothermal flash was carried out for comparison.

To compare the results it is necessary to clarify the implementation details. The Wilson K-factor was used for initialisation, with an aqueous phase introduced if water was present in the example as described by equations 29 and 30. Following initialisation 5 partial Newton steps were used, with phases removed where necessary. If oscillations were detected then a new phase was introduced if possible or a switch to Q-function maximisation made. The solution was found using the full Newton method, with a limit of $(F) \times 10$ steps before it was deemed a failed point and a switch made to Q-function maximisation, where each step is counted as the evaluation of Newton step.

For isothermal flash the same Wilson K-factor approximation was used at the solution temperature to the isenthalpic flash. The initial estimate was improved using 3 steps of successive substitution followed by a switch to a second order minimiser. For two phases the restricted step method described by ${ }^{\text {}}$, chapter 10 was used. For the multiphase case there is no ideal scaling factor, there are various possible implementations, often with Murray's method of lines preferred. Here we chose to use a trust region implementation with the

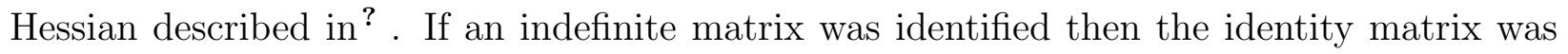
added with a scaling factor suitabable to ensure positive definiteness of the Hessian, a trust 
region method was used to guarantee convergence towards a local minimum.

Each method was implemented in FORTRAN and the wall clock time recorded along with the number of iterations. The results for each of the considered examples is given in table 3. Example 7 is not included as there is no 3 -phase region in $(P, T)$ space. The cost of each method is given relative to the cost of the isothermal flash. A switch to Q-function maximisation was necessary in less than $1 \%$ of the cases.

The phase envelope of example 3 is shown in figure 5 with the number of isothermal and isenthalpic flash iterations. This example is a relatively simple natural gas, it is relatively narrow boiling but would not present significant challenges when solved using a nested isothermal flash. In total isenthalpic flash required 1.07 times as many iterations as isothermal flash. Examples 2-6 required between 1.05 - 1.3 times as many iterations for isenthalpic flash than isothermal flash over the full phase envelope.

The number of second order iterations required for $(P, T)$ and $(P, H)$ flash is small over most of the phase envelope. In the close to critical region both isothermal and isenthalpic flash require more iterations, though in total only a tiny fraction (0.01\%) require a switch to Q-function maximisation.

It is noticeable that outwith the two-phase region isothermal flash uses no iterations while isenthalpic flash often requires a small number. This is because with isothermal flash the Gibbs energy of the feed mixture composition can be compared with the initial estimate from the Wilson K-factor approximation and the estimate discarded if the two-phase Gibbs energy is greater than the single phase Gibbs energy. However this is not possible in isenthalpic flash, which must continue until the phase is removed by an iteration of the partial Newton method, which in some cases uses a small number of full Newton steps before it is clear that the phase can be safely removed.

Although table 3 indicates that the cost of Q-function maximisation is between 5-6 times greater than $(P, T)$ flash, if the stability analysis used is tuned for the mixture then the cost can be significantly reduced. For example 3 it is necessary only to carry out stability analysis 
for one gas and one liquid estimate (e.g. from the Wilson K-factor approximation). Doing this reduces the cost for this example from 5.39 to 3.1 times greater than isothermal flash. Similar results are found for examples 2, 5, and 6 .

Example 4 is a narrow boiling mixture, it is therefore difficult to solve using a nested isothermal flash. This can be viewed in table 3. Close to the bubble curve the phase fraction of vapour changes very rapidly from 0 to 0.99 in $(P, T)$ space as the methane component evaporates. In this region the Hessian of the Q-function (equation 27) changes rapidly with the temperature (and is discontinuous at the phase boundary) in a manner similar to that shown in figure 1 for example 2. This leads to over-steps, or under-steps increasing the number of iterations necessary. The phase envelope and number of iterations required for $(P, T)$ flash and $(P, H)$ flash is given in figure 6.

Solving the isenthalpic flash problem directly for this mixture is often successful. The mixture is narrow boiling in that for a very small change in temperature there is a very large change in enthalpy, however this is not a problem if we specify the enthalpy. A large change in enthalpy will only lead to a relatively small change in temperature and the problem can be solved quite easily. The additional cost of isenthalpic flash is not significantly different from the other mixtures for this narrow boiling example. For the Q-function maximisation oversteps were common and often it was necessary to use a fully robust implementation with stability analysis carried out at every iteration.

Example 5 is a mixture containing water, this example is very similar to example 2 but with less components. This mixture contains a heavy oil pseudo-component and a light gas pseudo-component along with two intermediate components, and is typical of what may be used for the simulation of thermal recovery with a light solvent component. The phase envelope is given in figure 7 . For this example the modified initialisation procedure including water was used.

Using the modified initialisation procedure ensures that the heavy oil mixtures with water can be dealt with without any additional difficulty. The only region with a significant 
increase in the number of iterations when compared with the isothermal flash is the close to critical VLE region. In this region the initial estimate may indicate multiple phases (in some cases 4 seperate phases are introduced), which must be removed before more accurate initial estimates are generated from stability analysis, and it is often necessary to switch to Q-function maximisation. Close to the VLLE to LLE and VLLE to VLE boundary there is not a significant change in the number of iterations.

Example 2 is similar to example 5 but with a larger number of components and less light components. Over the full phase envelope the conclusions are the same as example 5, with difficulty only in the close to critical region. However table 3 shows that the computational cost is much closer to that for isothermal flash. This is in part due to the increased number of components, the dominant term in the computational cost is the decomposition of the Jacobian (or Hessian) in this example. These are almost of the same size and therefore differ only slightly in cost. Though the additional cost compared to isothermal flash is moderate, the use of 20 components is often considered prohibitively expensive for reservoir simulation.

A more complex system is considered in example 6. This is not relevant to heavy oil reservoir simulation but does test the generality of the implementation for isenthalpic flash. Up to four phases can co-exist at low temperatures. The hydrogen sulphide, carbon dioxide and methane can each form a separate nearly pure phase along with a vapour phase. The region where up to four phases are in equilibrium is presented in figure 8 along with the number of iterations for convergence with the presented implementation of isenthalpic flash.

The initialisation was based on the two phase Wilson K-factor approximation. To introduce each of the remaining liquid phases it was necessary to carry out stability analysis. Without a good initial estimate for each of the new phases there is a significantly larger number of switches to Q-function maximisation (10\% in this example). The region where this happened most often is visible in figure 8 as the region where more than 15 iterations were often necessary. Furthermore there are a larger number of cases where, following initialisation the phases oscillate between liquid and vapour, causing an immediate switch to 
Q-function maximisation. This leads to the patched appearance of figure 8. Table 3 presents the computational time over the region of 1 bar $<P<120$ bar and $-2000 \mathrm{~K}<H / R<1000 \mathrm{~K}$ where the cost, and number of switches to Q-function maximisation, was similar to other mixtures. Over the region presented in figure 8 the computational cost of isenthalpic flash was 2.3 times the cost of isothermal flash.

Example 7 is an equimolar mixture of water and n-butane. Often this mixture can have more phases present in equilibrium than components. The $(P, H)$ phase envelope for the mixture is given in figure 9, this figure also shows the number of second order isenthalpic flash iterations necessary to solve the phase split calculation.

Even in the region where there are more components than phases there are no significant problems encountered using the proposed methods. Q-function maximisation was used in less than $0.1 \%$ of cases. There are an increased number of iterations at the boundary between VLE and LLE and the boundary between two and three phases. This is often due to the introduction and removal of phases. When the initialisation, or stability analysis, indicated that more phases than components were necessary the type of root desired from the equation of state was selected. This is a simple and effective way to deal with the case where there are more phases than components.

\section{Conclusion}

Multiphase isenthalpic flash is important to process and reservoir simulations, and particularly useful for simulating thermal recovery of heavy oil. Calculation of multiphase isenthalpic flash is challenging since it needs to handle both isenthalpic flash and multiple phases. There is a strong need for reliable and efficient algorithms to calculate multiphase isenthalpic flash. This study provides two types of solutions for multiphase isenthalpic flash with full thermodynamics and with ideal solution thermodynamics, respectively. For the former situation, we present a general approach using the partial Newton and full Newton methods to solve 
the majority of cases efficiently and the Q-function maximization method to safeguard convergence if the Newton methods fail. We also show how to adjust the solution approach for heavy oil-water systems encountered in thermal recovery simulation. For the latter situation, we propose a simple formulation as the extension of the multiphase isothermal flash for ideal solution systems. The formulation can be solved with Newton's method for efficiency while the rare divergent exceptions can be handled with a nested loop approach that converges the temperature in the outer loop.

The proposed solution approaches are tested with 7 examples over a wide pressure and molar enthalpy range. Both the solution approach for ideal solution thermodynamics and the general approach for full thermodynamics can solve their respective examples without failure. In particular, the general approach proves successful in difficult cases including a narrow boiling system, a complex multiphase system with up to four non-aqueous fluid phases, and a degenerate system with 3 phases and 2 components.

The extensive tests also provide the computation speed of the proposed multiphase isenthalpic flash procedures relative to their corresponding multiphase isothermal flash procedures. For the ideal solution case (example 1), multiphase isenthalpic flash is around 3 times slower. Since the ideal solution flash often does not dominate thermal reservoir simulation costs, the proposed approach should be efficient enough. For the other cases with full thermodynamics, the cost of isenthalpic flash using the general approach is 1.1 to 2.3 times that of isothermal flash. It is a modest increase in computation time, indicating that compositional simulation of thermal recovery with full thermodynamics is not prohibitive. It should be noted that the computational costs in this study are for "blind" flash where no previous results are used to initialize the flash. This is different from the situation in reservoir simulations where the previous results can usually provide good initial estimates for direct Newton iterations. It requires a dedicated study to evaluate the relative speed of isenthalpic flash in simulations. Nevertheless, it can be expected that the relative speed is more dominated by the full Newton step. 
For multiphase isenthalpic flash specific to thermal recovery simulation where there can be a third aqueous phase in addition to the gas and oil phases, it is recommended to introduce the aqueous phase during the intialisation. This makes the method convergent in a larger number of examples without resorting to Q-function maximisation. The cost of threephase isenthalpic flash is very similar to three-phase isothermal flash using this initialisation method.

Finally, although the focus of this paper is on multiphase isenthalpic flash, the methods discussed can be easily extended to multiphase flash with other state function based specifications (e.g. $(P S),(V, T),(V, U)$ and $(V, S))$.

\section{Notation}

The use of bold for a lower-case letter indicates that symbol represents a vector and a bold uppercase letter represents a matrix.

\section{Greek letters}

$\alpha \quad$ Step length modifier

$\beta \quad$ Phase mole fraction

$\hat{\varphi} \quad$ Fugacity coefficient

$\omega \quad$ The acentric factor

\section{Latin letters}

$\hat{f} \quad$ Fugacity

C Number of components

$C_{p} \quad$ Heat capacity

E Variable defined in equation 2 
F $\quad$ Number of phases

$G \quad$ The Gibbs energy

$g \quad$ Equation to be solved at equilibrium

$H \quad$ Enthalpy

$h \quad$ Component enthalpy

$J \quad$ Jacobian of a system of equations

K equilibrium K-factor

$n \quad$ mole number

$P \quad$ Pressure

$Q \quad$ Function which is at a minimum (or maximum) at the solution

$R \quad$ Universal gas constant

$S \quad$ Entropy

T Temperature

$U \quad$ Internal energy

V Volume

y component mole fraction

$z \quad$ Feed mole number

\section{Subscripts}

c Critical point property

i Component index 
j Phase index

$k \quad$ Component index

$l \quad$ index for equation 20 and 18

$m \quad$ Phase index

min Minimum

new Value following update

o $\quad$ index for equation 20 and 18

$w \quad$ Water component

\section{Superscripts}

$\begin{array}{ll}a & \text { Aqueous phase } \\ I G & \text { Ideal gas } \\ l & \text { Liquid phase } \\ r & \text { residual } \\ \text { spec } & \text { Specified variable } \\ v & \\ v & \text { Vapour phase }\end{array}$




\begin{tabular}{c|c|c} 
& PH Flash & Recommended methods \\
\hline 1 & Correlations for K-factors (and heat & Ideal solution approach \\
& capacities) & Partial Newton \\
\hline 2 & EoS or other full thermodynamic model & Full Newton \\
& Q-function maximisation \\
\hline 3 & Same models as 2 for gas-oil-aqueous & Adaption of 2 for initialisation and \\
& systems in thermal recovery & stability analysis
\end{tabular}

Table 1: An overview of the $\mathrm{PH}$ flash problems studied in this work

\begin{tabular}{l|l|l|l|l} 
& Components & Max phases & System description & Model \\
\hline Example 1 & 7 & 3 & synthetic oil and water & $\mathrm{IS}$ \\
Example 2 & 20 & 3 & heavy oil and water & $\mathrm{PR}$ \\
Example 3 & 7 & 2 & natural gas & $\mathrm{SRK}$ \\
Example 4 & 2 & 2 & $\mathrm{C}_{1} \& \mathrm{nC}_{4}$, narrow boiling & $\mathrm{PR}$ \\
Example 5 & 5 & 3 & oil and water & $\mathrm{PR}$ \\
Example 6 & 5 & 4 & $\mathrm{C}_{1}, \mathrm{C}_{2}, \mathrm{C}_{3}, \mathrm{CO}_{2}, \mathrm{H}_{2} \mathrm{~S}$ & $\mathrm{SRK}$ \\
Example 7 & 2 & 3 & n-butane and water & $\mathrm{PR}$
\end{tabular}

Table 2: Summary of mixtures considered in this work

\begin{tabular}{l|l|l} 
Mixture & \multicolumn{2}{|l}{ Cost relative to $(P, T)$ flash } \\
& $(P, H)$ Flash & Q-Function \\
\hline Example 2 & 1.12 & 3.77 \\
Example 3 & 1.65 & 5.39 \\
Example 4 & 1.75 & 10.84 \\
Example 5 & 1.63 & 5.64 \\
Example 6 & 1.71 & 6.34
\end{tabular}

Table 3: Computational cost comparison of $(P, H)$ and $(P, T)$ flash 


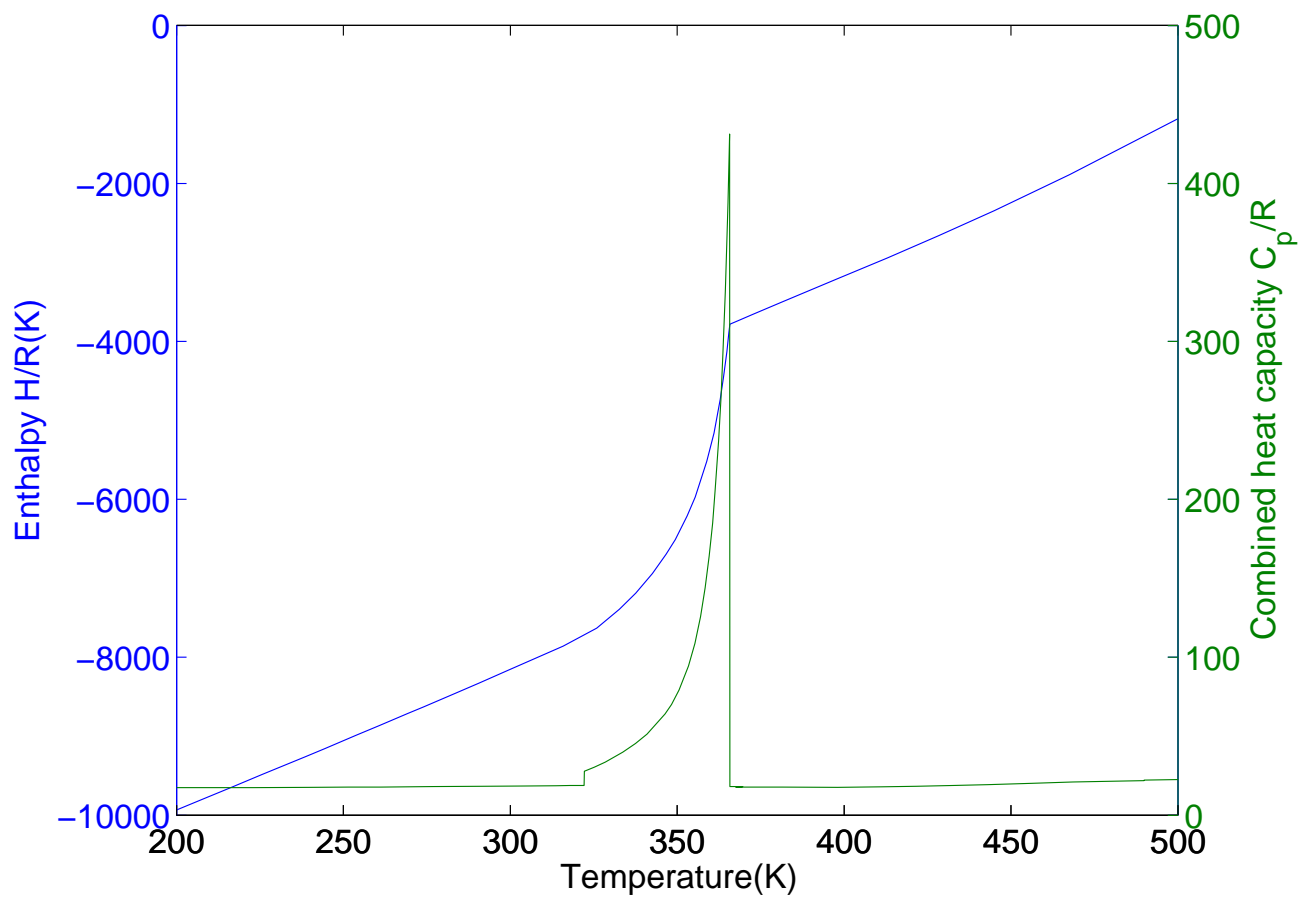

Figure 1: The enthalpy and combined heat capacity (of both the pure phases and of phase change), for example 1 between $200 \mathrm{~K}$ and $500 \mathrm{~K}$ at 1 bar. Equation 27 is $-T^{2} \times$ the combined heat capacity. Change from LLE to VLLE at $322.1 \mathrm{~K}$ and from VLLE to VLE at $365.8 \mathrm{~K}$ correspond to the discontinuities in the combined heat capacity. 


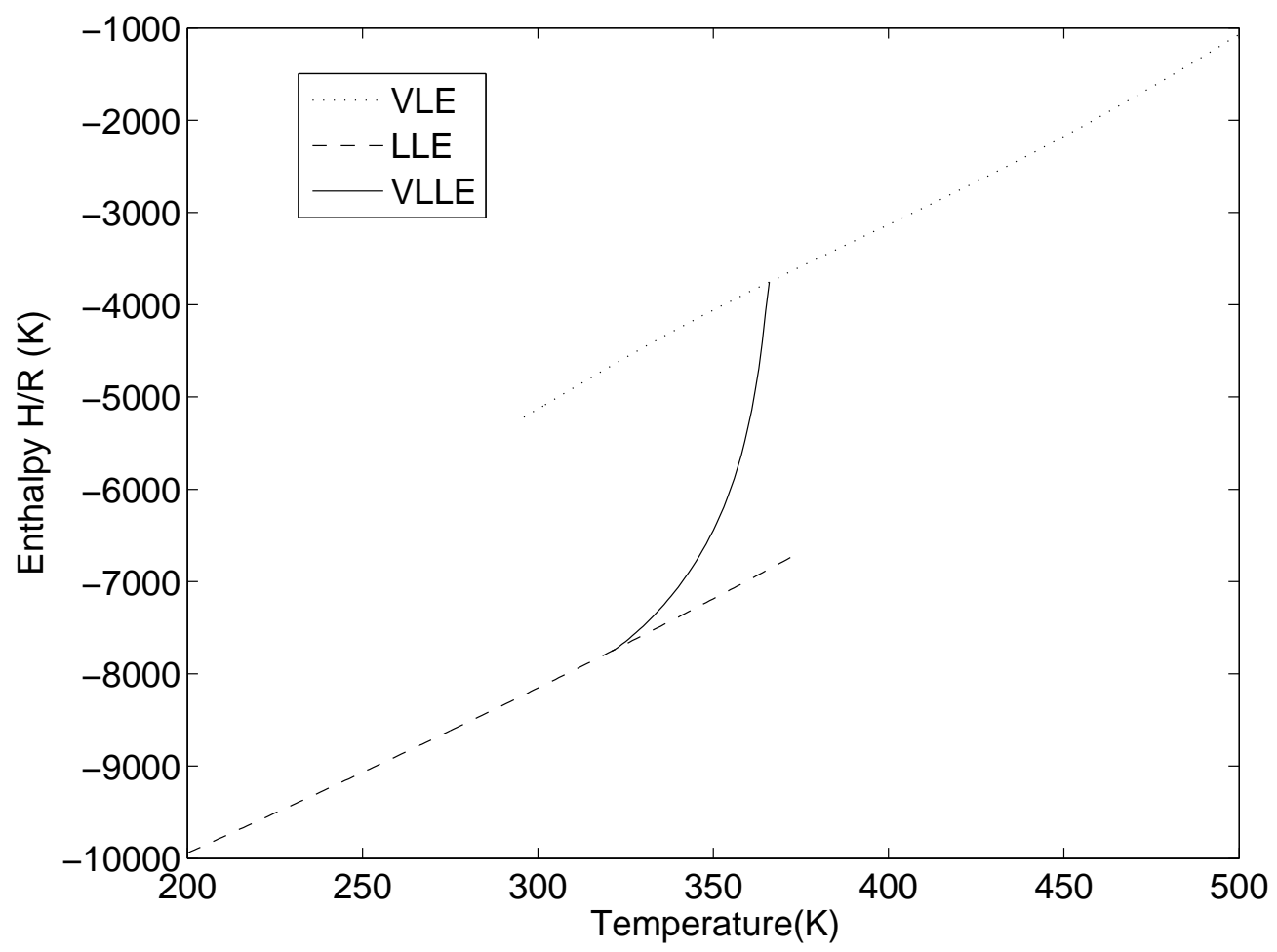

Figure 2: The enthalpy of the VLE, LLE and VLLE solutions to the isothermal flash at 1 bar between $200 \mathrm{~K}-500 \mathrm{~K}$ for example 1 . It is not possible to meet an enthalpy constraint between $\mathrm{H} / \mathrm{R}=-5175 \mathrm{~K}$ and $\mathrm{H} / \mathrm{R}=-6700 \mathrm{~K}$ with only two phases.

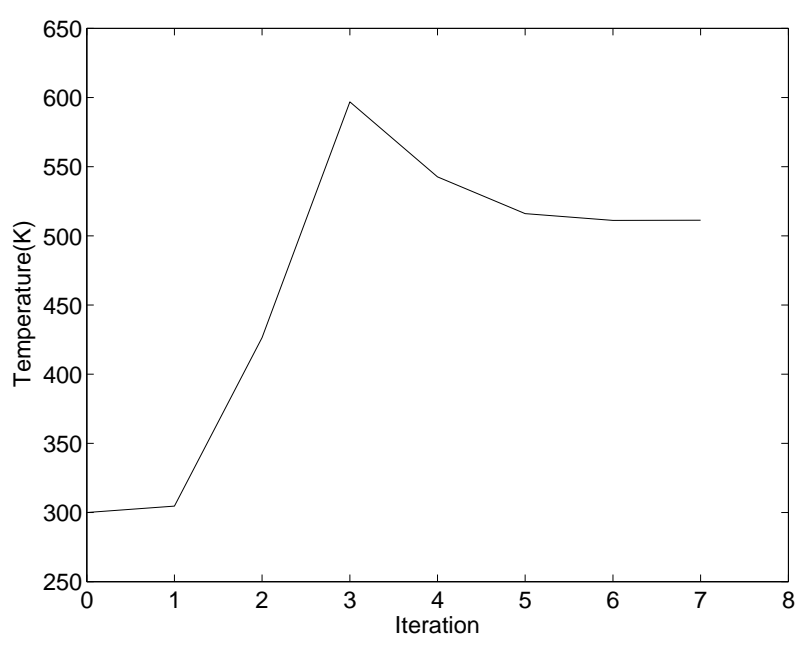

(a) Temperature convergence

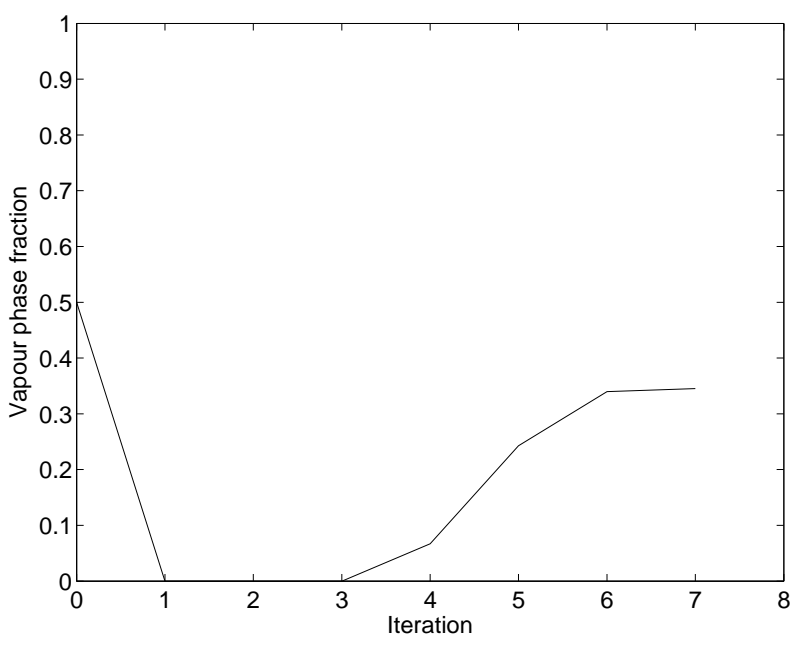

(b) Vapour fraction convergence

Figure 3: Convergence for ideal solution approximation of example 1. Initial estimate of $\beta=0.5$ and $T=300 K$ and a solution of $\beta=0.345$ and $T=511.2 K$. 


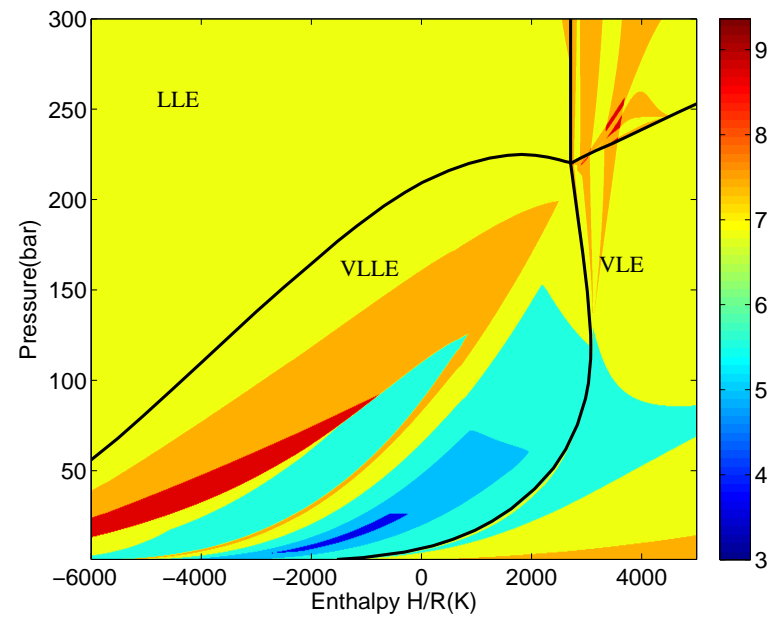

(a) $(P, T)$ flash iterations

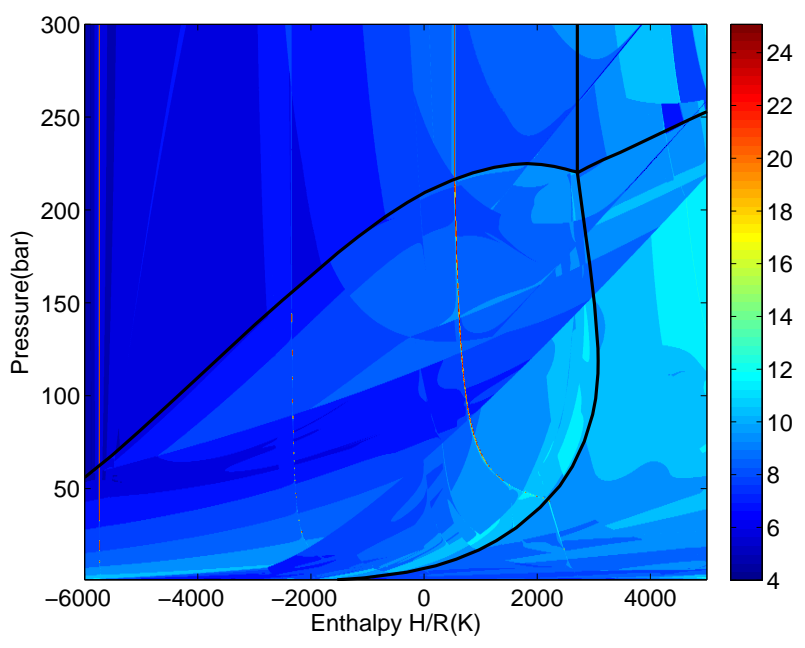

(b) $(P, H)$ flash iterations

Figure 4: Comparison of the number of iterations necessary to solve the isenthalpic and isothermal flash for example 1. The colour indicates the number of iterations required to find the solution, with different scaling on the colours used.

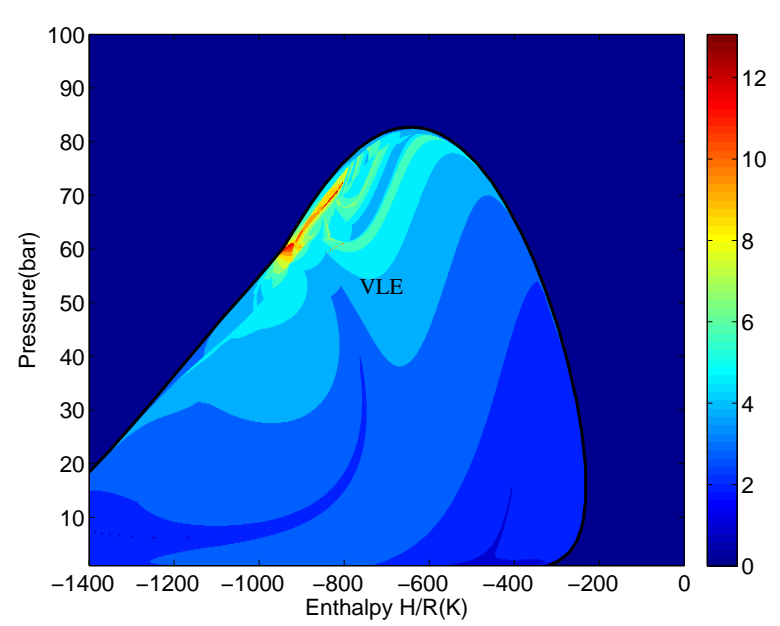

(a) $(P, T)$ flash iterations

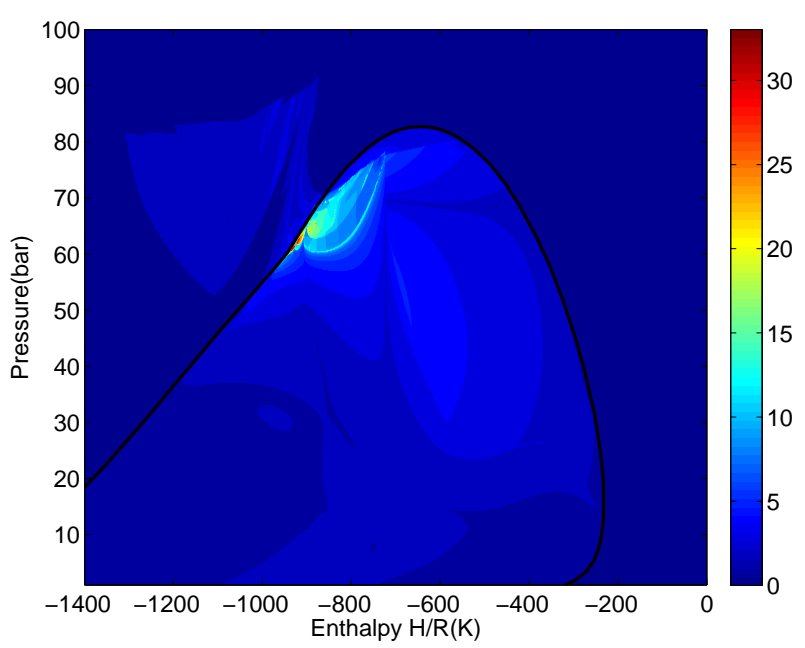

(b) $(P, H)$ flash iterations

Figure 5: Comparison of the number of iterations necessary for isothermal flash and isenthalpic flash for example 3. The iteration counter is for only the second order method used. 


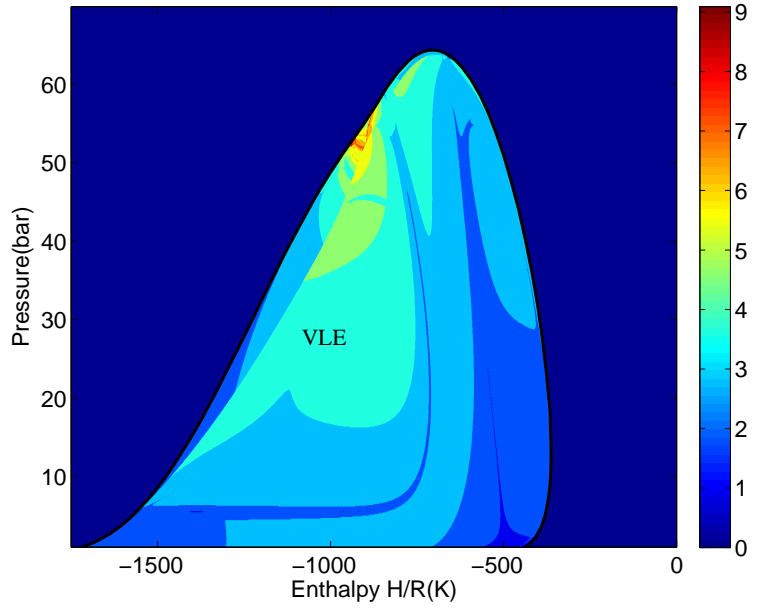

(a) $(P, T)$ flash iterations

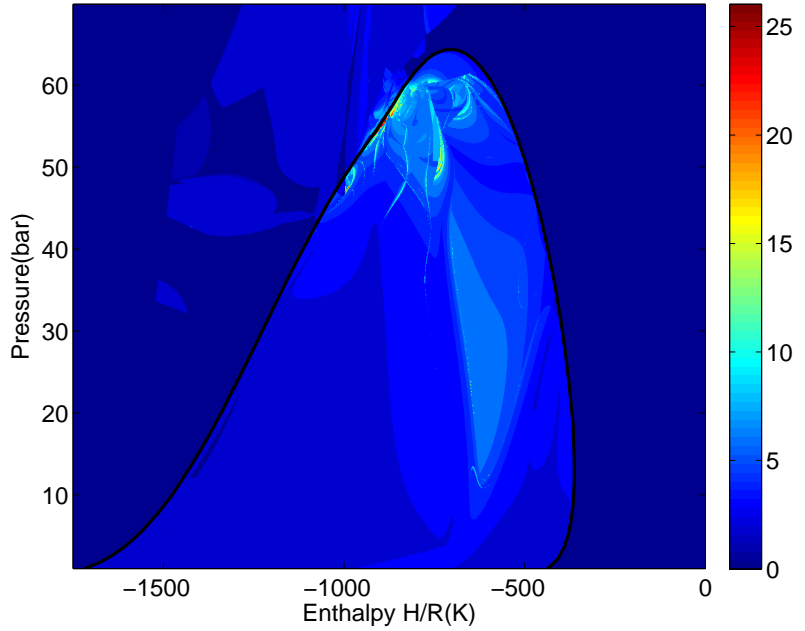

(b) $(P, H)$ flash iterations

Figure 6: Comparison of the number of iterations necessary for isothermal flash and isenthalpic flash for example 4 . The iteration counter is for only the second order method.

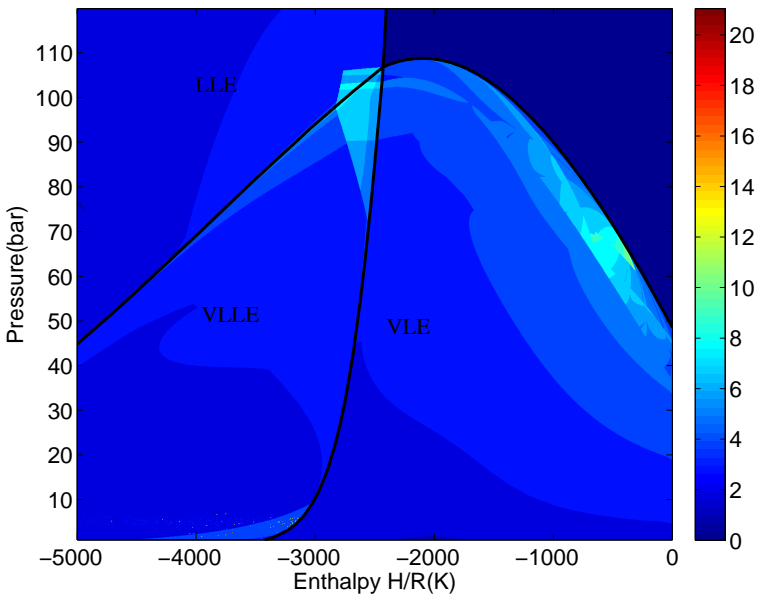

(a) $(P, T)$ flash iterations

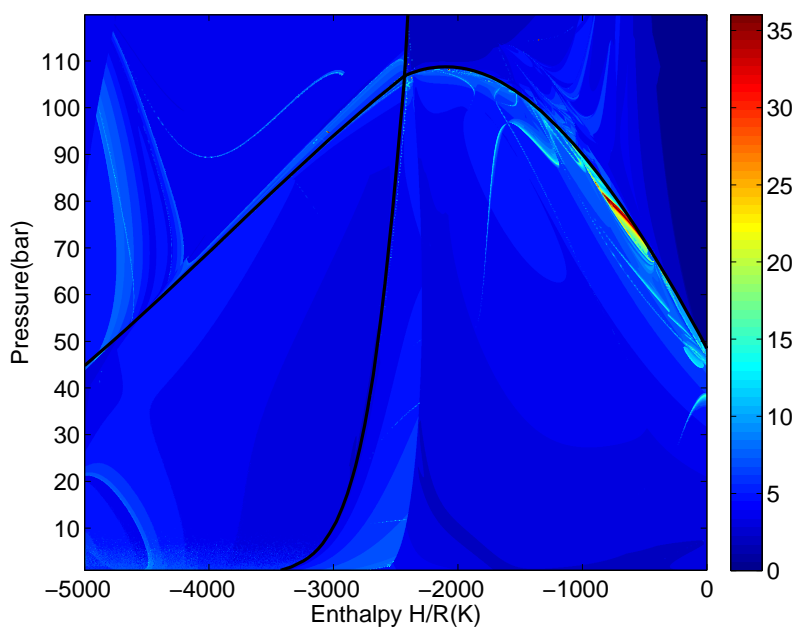

(b) $(P, H)$ flash iterations

Figure 7: Comparison of the number of iterations necessary for isothermal flash and isenthalpic flash for example 5. The iteration counter is for only the second order method. 


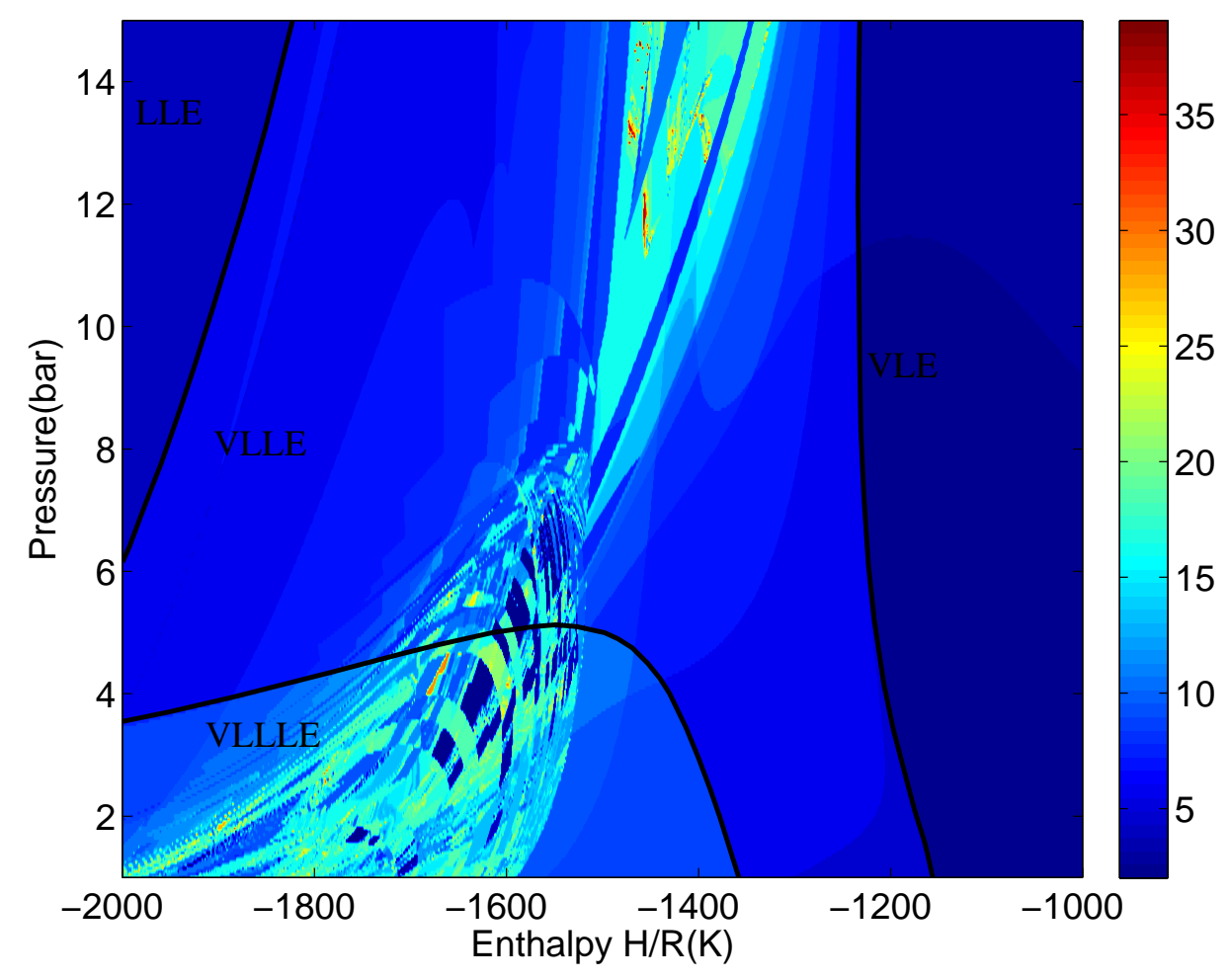

Figure 8: Phase envelope and number of $(P, H)$ flash iterations necessary. The enthalpy range corresponds to temperatures between $110 \mathrm{~K}$ and $200 \mathrm{~K}$. The backup Q-function maximisation was necessary in $10 \%$ of cases. 


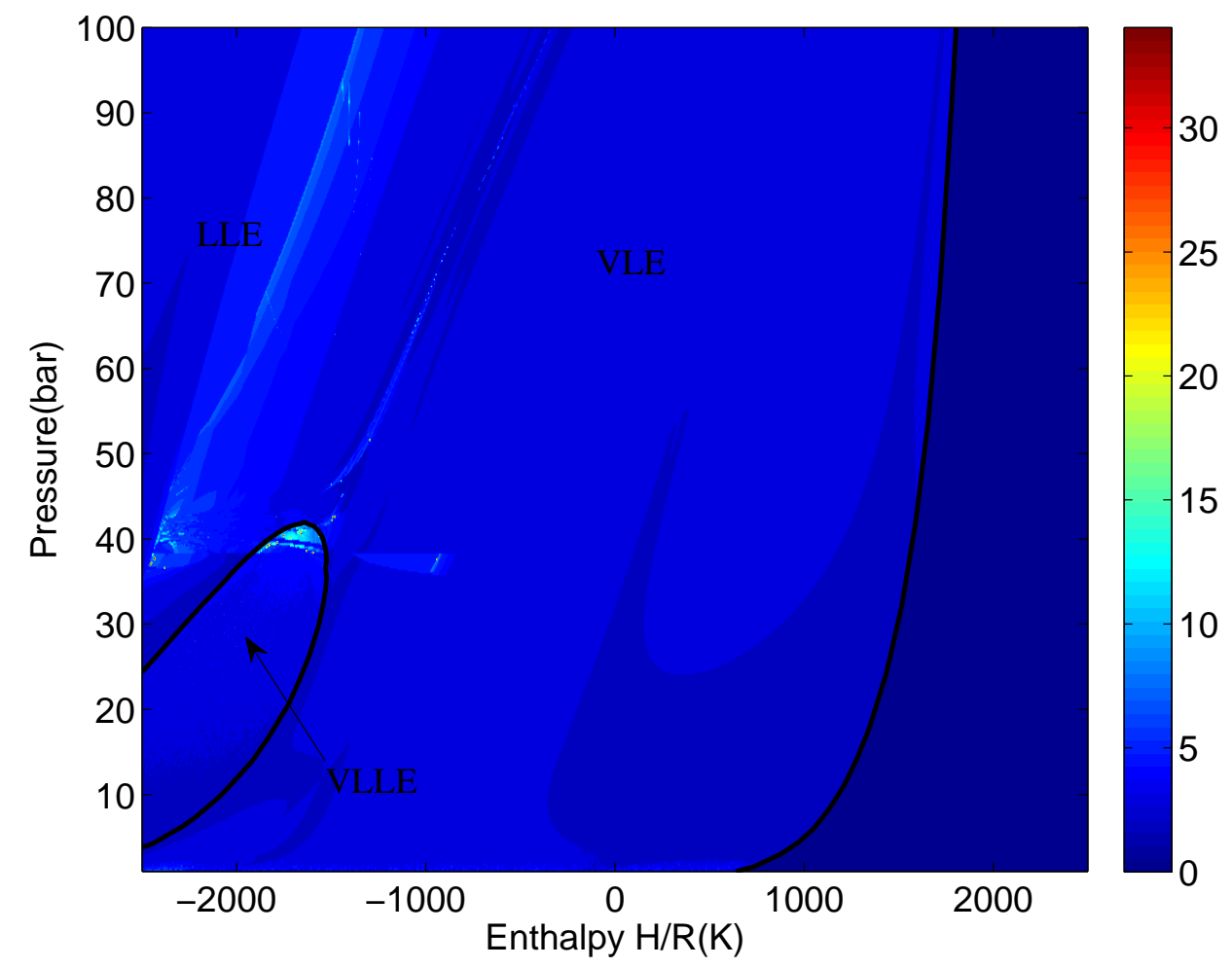

Figure 9: Heat plot of the number of second order iterations necessary to solve the phase split problem for an equimolar mixture of water and butane. The three phase region corresponds to a discontinuity in the enthalpy in $(P, T)$ space. The $(P, H)$ region scanned corresponds to a temperature range of $300 \mathrm{~K}$ to $580 \mathrm{~K}$. 